\title{
Ethnicité et nouveaux mouvements sociaux au Cameroun
}

\begin{abstract}
Georges Macaire Eyenga ${ }^{1}$
[Résumé] L'institutionnalisation des nouveaux mouvements sociaux (NMS) en Afrique subsaharienne a donné à l'anthropologie sociale et politique, un champ d'étude pertinent permettant d'appréhender les mobilisations collectives en contexte de démocratisation. Cette appréhension doit se faire en travaillant une méfiance envers les approches occidentalo-centriques de l'étude des mobilisations. Nous avançons comme idée principale que l'anthropologie des mobilisations en Afrique subsaharienne doit considérer l'ethnicité comme une technologie culturelle structurant la formation et l'action des NMS. Cette réflexion porte particulièrement sur le Cameroun qui présente en son sein de très nombreux NMS donc l'action collective ne peut être saisie sans la prise en compte de la variable ethnique. II ressort de cette étude deux constats : la formation des NMS sur la base de l'ethnicité de la période précoloniale à celle postcoloniale, et la prédominance du discours ethnique sur le discours nationaliste dans les mobilisations sociales.
\end{abstract}

Mots-clés : ethnicité, nouveaux mouvements sociaux, minorités, champ politique, mobilisations, Cameroun.

[Abstract] The institutionalization of new social movements (NSM) in Africa has provided a relevant field of study to social and political anthropology that helps to understand collective mobilizations in a context of democratization. It is about understanding the significant expressions of African new social movements by being nevertheless careful about Western-centric approaches in the study of mobilizations. The main idea here is that the anthropology of mobilizations in Sub-Saharan Africa should consider ethnicity as cultural technology that structure the formation and the action of new social movements. This study particularly focuses on Cameroon, a Central African country with many new social movements whose collective action cannot be understood if their ethnic dimension is not considered. Two main conclusions of this study are: the formation of new social movements based on the ethnicity from the pre-colonial period to the post-colonial period and the preponderance of the ethnic discourses over the nationalist ones in social mobilizations.

Keywords: ethnicity, new social movements, minorities, political field, mobilizations, Cameroon.

1 Doctorant en Sociologie à l'Université Paris Ouest Nanterre La Défense.

L'auteur remercie vivement Cindy Morillas, Mbebi Raymond et Bobo Bobo R. Faustin pour leurs commentaires sur cet article. II remercie aussi Nji Ibrahim Mouiche pour ses conseils sur l'approche socio-anthropologique des (nouveaux) mouvements sociaux en Afrique subsaharienne. 


\section{Introduction}

Les nouveaux mouvements sociaux (NMS ${ }^{2}$ ) renseignent sur les particularités de la vie socio-politique de l'Afrique subsaharienne, à l'instar du processus de démocratisation amorcée depuis les années 1990, de la crise économique, de la dégradation des conditions de vie des populations, de l'instabilité politique, de la recrudescence des conflits intra-étatiques et de la montée du religieux (Copans, Buijtenhuijs, 1982 : 4). Leurs trajectoires sur le terrain africain traduisent une dynamique commune à toute institution évoluant dans un écosystème en mouvement.

Dans les expériences classiques de démocratie, il existe une énorme littérature sur les mobilisations et les mouvements sociaux. Par exemple, de nombreuses études sur l'action collective offrent des connaissances pertinentes pour rendre intelligible la compréhension de cet objet d'étude. Pour Donatella della Porta et Mario Diani (2006), les NMS sont des réseaux informels d'acteurs collectifs et individuels fondés sur des croyances partagées et des pratiques de solidarité orientées vers la protestation. Cette définition fera très vite l'objet d'une pluralité de contestations, notamment par l'école de la mobilisation des ressources. Des auteurs comme David S. Meyer (2002) et Sydney Tarrow (2005) soutiennent que les NMS entrent en interaction avec l'État ou ses institutions. Les tenants d'une approche interactionniste récusent l'idée du conflit dans l'expression des NMS.

En outre, il faut différencier les NMS des partis politiques perçus comme des acteurs de la politique institutionnelle, intervenant au sein de l'arène électorale (Dufour, Traisnel, 2009: 39). Dans le cadre de cette étude, nous partageons l'approche interactionniste qui analyse l'action des NMS par rapport à la position qu'occupent leurs minorités ethniques dans le système politique. Cette approche permet de mettre l'accent sur la dimension instrumentale des NMS et sur l'usage des moyens conventionnels, institutionnels et pacifiques. Au Cameroun, les NMS que nous avons étudiés ne s'opposent pas au système politique établi, mais visent plutôt à l'intégrer, à participer à la gestion des affaires publiques (Mbebi, 2015: 13). En tout état de cause, les NMS désignent dans notre étude des associations qui se voulaient au départ apolitiques, mais qui en viennent à se politiser au fil de leurs développements et relations avec les instances étatiques (Arcand, 2003: 32-33). Ils sont le fait des minorités ethniques qui cherchent à entrer dans le système politique afin d'accéder aux instances décisionnelles (Lapeyronnie, 1988 : 595-602).

Contrairement à ceux d'Amérique latine ou d'Europe de l'Ouest, les NMS d'Afrique Subsaharienne ne se sont pas encore vus consacrer une large production scientifique. Cette faiblesse est difficile à expliquer dans la mesure où cette partie du continent a longtemps été le lieu d'importantes formes de mobilisations sociales et politiques. Les

2 Dans le cadre de ce travail NMS désigne «nouveaux mouvements sociaux». 
mouvements sociaux dans des pays comme le Gabon, la République du Congo, le Cameroun, le Tchad ou la Centrafrique ont été, lors de la libéralisation de leur vie politique, des acteurs de la résistance contre les gouvernements autoritaires. De plus, cette partie de l'Afrique connaît depuis environ deux décennies un développement remarquable de sa société civile et des groupes de pression.

Cependant, la rareté des études sur les NMS en Afrique subsaharienne ne signifie pas l'inexistence d'efforts scientifiques en la matière. Sauf que, pour étudier les NMS sous les tropiques, de nombreuses préoccupations épistémologiques émergent. Doit-on appliquer à cet espace les mêmes cadres d'analyses usités pour l'étude des NMS en Europe ? À cette question, Nathalie McSween (2010) réplique qu'il faut dépasser tant les approches s'articulant autour du concept de société civile que celles qui utilisent le modèle du processus politique, pour la simple raison que ces approches arrivent difficilement à expliquer ces nouveaux phénomènes en Afrique. En effet, l'approche par la société civile, en gardant avec elle l'idée occidentale de la "société civile», définie par John Locke et Hegel, exclut d'emblée les associations informelles et celles qui étaient considérées comme "non-civiques », telle que les associations basées sur l'adhésion ethniques ou religieuses. En Afrique subsaharienne, cela reviendrait à exclure la majorité des mobilisations, puisqu'on y retrouve de nombreux mouvements fondés sur l'ethnie, la tribu, la langue et la religion. Par la suite, l'approche du processus politique est vivement critiquée par le courant culturel (Touraine, 1984) qui, sans réfuter ses résultats, affirme que cette approche privilégie davantage les facteurs structurels au détriment des facteurs non-structurels dans l'explication des mobilisations. Nathalie McSween (2010) suggère alors de déplacer le regard scientifique sur les NMS pour les considérer non plus en tant qu'organisations (de mouvement social ou de la société civile), mais en tant qu'épisodes de contestation. Cette idée permet de prendre en compte les logiques ethniques et d'allégeances patrimoniales dans les sociétés africaines. C'est largement dans cette perspective analytique que notre réflexion s'inscrit en essayant d'y contribuer davantage.

De ce fait, l'étude des NMS au Cameroun doit être objectivée par la mise en évidence de grilles d'analyse appropriées au contexte anthropologique du terrain d'observation. Cette réflexion sur les NMS dans cette société multiethnique impose de saisir un « référentiel global et local » (Muller, 2015) qui oriente l'engagement politique, économique et culturel des entrepreneurs sociaux. Le communautarisme ethnique que l'on retrouve chez les groupes sociaux en Afrique subsaharienne nécessite que l'on considère l'ethnicité comme une technologie culturelle pertinente d'identification des NMS. C'est que le contexte ethnique constitue une dimension essentielle des modes d'organisation et de perception de soi en Afrique puisque, sur le plan historique, «c'est le cadre ethnique qui a toujours assuré la structure fondamentale de l'héritage culturel, spirituel et artistique des populations africaines » (Mouiche, 2000 : 47). Le concept d'ethnicité a

3 Le Cameroun compte environ plus de 240 groupes ethniques différents. 
été largement développé en socio-anthropologie où il est présenté comme un élément culturel et identitaire de l'individu, se plaçant sur le terrain des interactions culturelles et sociales (Weber, 1978 ; Eriksen, 1991 ; Fenton, 2010). Selon Marco Martinello (1995), l'ethnicité se définit comme l'une des formes majeures de différenciation sociale et politique, d'une part, et d'inégalité structurelle, d'autre part. Elle repose sur «la production et la reproduction de définitions sociales et politiques de la différence physique, psychologique et culturelle entre les groupes dits ethniques qui développent entre eux des relations de différents types (coopération, conflits, compétition, domination, reconnaissance, etc.) 》(Martinello, 1995 : 18-19).

En mettant en exergue l'ethnicité dans les attitudes et les comportements sociopolitiques, nous n'avons aucunement l'intention de survaloriser la charge ethnique. Il n'est non plus question d'étudier de manière fonctionnelle l'organisation de ces NMS. Il s'agit bien plutôt de révéler un fait dont la permanence ne saurait troubler l'acuité du regard scientifique. Étudier ce phénomène, c'est se placer au cœur de l'une des réalités importantes de l'histoire africaine (Njoya, 2002 : 240). Par conséquent, nous récusons l'idée d'un déterminisme absolu de l'ethnicité sur les NMS au Cameroun. Le socle ethnique des NMS est lié à la position marginale des groupes ethniques dans le système politique camerounais. C'est pourquoi, ces NMS dont les actions prennent sens dans l'ethnicité sont souvent des acteurs stratégiques du positionnement politico-administratif des minorités ethniques. L'ethnicité devient pour ces NMS une technologie culturelle utile pour leur formation et leur l'entrepreneuriat. Dès lors, cet article envisage de faire une anthropologie des mobilisations en deux points. D'une part, montrer que l'instrumentalisation de l'ethnicité en contexte d'ouverture politique au Cameroun a permis à certaines minorités ethniques de se constituer en NMS. D'autre part, analyser la manière dont le discours ethnique supplante celui nationaliste dans l'activation de l'action collective au Cameroun.

\section{La constitution ethnique des NMS au Cameroun}

Longtemps, les recherches qui étudiaient l'Afrique faisaient montre d'une certaine réticence à évoquer l'ethnicité à propos de ce continent, de peur d'alimenter les préjugés raciaux de leurs lecteurs (Lonsdale, 1996: 38). Mais cette réticence est aujourd'hui évacuée avec plus de facilité dans la mesure où l'ethnicité n'est plus perçue, du moins par un bon nombre de chercheurs africanistes, comme un élément dangereux pour l'ordre social. Le recours à la ressource ethnique est d'ailleurs une pratique fréquente dans la formation des partis politiques au lendemain des indépendances et lors de la libéralisation politique. Bien que proscrite dans la Constitution ${ }^{4}$ du Cameroun, la formation des partis politiques sur une base ethno-communautaire est une réalité incontestable.

4 Cf. les articles 3 de la Constitution du 2 juin 1972, et de la loi n 96/06 du 18 janvier 1996 portant révision de la Constitution du 2 juin 1972 et la loi n 90/056 du 19 décembre 1990 relative aux partis politiques (article 1). 
L'ethnie est instrumentalisée à des fins de calcul politique en vue de l'occupation des positions de pouvoir dans l'ordre dirigeant (Menthong, 1998: 43). La place de choix qu'occupe l'ethnicité dans les formations partisanes se retrouve aussi dans celles des NMS. L'analyse de la constitution ethnique NMS au Cameroun passe par l'historicité du recours à l'ethnie dans les mobilisations et par l'objectivation des expressions ethniques des NMS en contexte de démocratisation.

\subsection{Historicité du recours à l'ethnie dans les mobilisations sociales}

Comme l'affirme l'anthropologue Ibrahim Mouiche (2000), en Afrique noire contemporaine, le fait ethnique est antérieur au fait étatique. Cet auteur voulait par-là signifier que l'ethnicité en tant que ressource d'identification et de mobilisation des organisations humaines possède une histoire très ancienne. En effet, plusieurs courants contemporains de l'anthropologie et de la sociologie ont déjà cultivé l'étude du mouvement social, et de sa variété, jusqu'aux sociétés précoloniales, et ils sont rejoints aujourd'hui par les historiens (Moniot, 2005 : 347). Avant la période coloniale, l'ethnie constituait essentiellement l'unité fondamentale des groupes humains. En plaçant un ancêtre unique au début d'une généalogie, le cadre ethnique devenait alors le cadre politique dont le groupe pouvait se revendiquer pour ses relations économiques, sociales et culturelles. Ce cadre recouvrait des enjeux liés aux besoins de sécurité du groupe ethnique pour son épanouissement (Olivier de Sardan, 1973 : 216). Les périodes de guerres interethniques pour le contrôle des chefferies, des ressources foncières, forestières et minières mobilisaient les armées à travers des alliances ethniques ou matrimoniales.

Dans la majeure partie des cas, l'époque coloniale fut brève : à peine plus d'un demisiècle. Elle a pourtant marqué les pays d'une empreinte indélébile, et légué des ferments de transformation profonde (Coquery-Vidrovitch, 2005: 413). Durant cette période, l'identité ethnique a continué à jouer un rôle important dans l'interaction avec les colonisateurs. L'ethnicité a été utilisée non seulement pour les luttes contre l'impérialisme colonial, mais aussi en tant qu'objet d'une instrumentalisation par le colonisateur pour mieux diviser et contrôler les territoires soumis (von Morgen, 1982). De plus, on constate que le moment colonial consacre, en effet, une sorte d'assimilation réciproque entre militaires, administrateurs civils et anthropologues généralistes autour d'un objectif commun : mieux connaître les sociétés conquises pour mieux les gouverner et fonder une "praxis coloniale» en établissant des liens « de causalité réciproque entre la connaissance et l'action » (Otayek, 2001 : 129-142). Ce constat peut pousser à croire que les anthropologues généralistes étaient alors au service du colonialisme. De nombreuses mobilisations sociales se sont ainsi produites durant la colonisation en s'exprimant par le biais d'une conscience identitaire ethnique. D'où la formule d'« anticolonialisme identitaire» utilisée ici pour désigner certaines formes ethniques d'anticolonialisme (Cahen, 2000). 
Selon Abel Eyinga (1985), la fin de la colonisation correspond aussi à l'émergence des partis politiques, ainsi que des mouvements ouvriers et syndicaux, bien ordonnés et structurés. Cependant, il faut préciser que ces mouvements, indigènes à la veille de l'indépendance, avaient tous l'allure de mouvements nationalistes alors qu'ils se formaient sur fond d'ethnicité (Dubois, Michel et al., 2000 : 319-333). De surcroît, ce réveil des mouvements sociaux a commencé au début des années vingt et s'est accentué au sortir de la crise de 1929-1930 en prenant corps dans une floraison d'associations à base ethnique, régionale et culturelle (Mbembe, 1986: 37). Cette précision est importante dans la mesure où cela permet de comprendre pourquoi pendant l'accession à l'indépendance - dans le cadre des mobilisations - c'est l'unité ethnique qui semblait prendre paradoxalement le pas sur la nation que les nouveaux dirigeants de l'État moderne cherchaient à construire en vain. L'amorce vers l'indépendance a réellement montré que l'ethnicité est l'un des répertoires privilégiés de mobilisations collectives et individuelles. L'esprit colonial était souvent méprisant au point de devenir insupportable à la fois pour les pouvoirs traditionnels qui voyaient leur avenir menacé et pour les «indigènes évolués » revenus des métropoles (Onana, 2004). C'est dans cette logique que vont se cristalliser les mobilisations des colonisés pour revendiquer l'institution d'une société prenant en compte la justice, l'égalité, le respect des identités et des cultures. C'est pourquoi les discours identitaires et la contestation de l'ordre colonial avaient ainsi viré en revendications politiques (Akindès, 2003 : 17).

Lorsque le Cameroun accède à l'indépendance en 1960, le président Ahmadou Ahidjo fut très vite confronté à la gestion d'un «État constitué de plus 240 groupes ethniques badigeonnant le paysage politique » (Njoya, 2001: 24). La plupart des mobilisations que l'on observa de 1960 à 1980 furent liées soit aux partis politiques dits d'opposition qui refusaient d'intégrer le parti « unifié» institué en 1966 à savoir l'Union camerounaise (UC) - soit aux minorités ethniques qui se sentaient lésées par la politique de l'équilibre régional mis en place par le pouvoir présidentiel afin de construire l'État unitaire. «La nature répressive de l'administration publique $~_{5}$ de cette période n'offrait malheureusement pas un environnement favorable à ces minorités pour exprimer leurs sentiments d'exclusion. En effet, la politique de l'équilibre régional et l'inefficience des partis politiques ont considérablement marginalisé les minorités ethniques au Cameroun. Elle a été contre l'inclusion sociopolitique des minorités Kirdi du GrandNord, des Haoussa, Mabi, Mankon et Maka-Bebend (Mbebi, 2015: 68-79). Comme les groupes démographiquement dominants ont été favorisés dans cette politique d'équilibre régional, l'accession des minoritaires aux instances décisionnelles restait difficile. Dès lors, l'avènement du processus de démocratisation dans le courant des années 1990

5 Cf. Achille Mbembe, "Le Cameroun après la mort d'Ahmadou Ahidjo », in Politique africaine, n

- 37, mars 1990. Voir aussi, Achille Mbembe, «crise de légitimité, restauration autoritaire et déliquescence de l'État», in P. Geschiere et P. Konings, Itinéraire d'accumulation au Cameroun, Paris, ASC-Karthala, 1993, p. 345-374. 
au Cameroun va créer un contexte permettant à ces minorités de se constituer en NMS sur fond d'ethnicité.

\subsection{Les expressions ethniques de la formation des NMS}

En Europe, les mouvements étudiants prenaient forme lorsque leurs sentiments de solidarité et d'indignation se mêlaient à la vie étudiante proprement dite et à une sensibilité plus ou moins forte des mouvements sociopolitiques (Dubet, 1994). Le cas le plus récent est sans doute la mobilisation en France, des lycéens et des étudiants contre la loi Travail'. À côté, les mouvements d'infirmiers étaient fondés sur leur rapport au travail (Hamel, 2008), tout comme ceux des policiers ou ouvriers d'usines des pays industrialisés. Les mouvements féministes (Jacquemart, 2011) et écologistes (Feenberg, 2005 ; Jacquiot, 2007) ont, entre autres, eu pour base de formation la citoyenneté ou le partage de certaines valeurs culturelles et protectionnistes qui s'éloignent de l'ethnicité. Cependant, en Afrique subsaharienne, la réalité apparaît tout à fait différente. De manière particulière, les NMS sur le continent noir sont pour l'essentiel — et non exclusivement - des minorités ethniques qui profitent des dispositions normatives (l'État de droit) et du contexte favorable de la démocratisation pour exprimer leurs revendications.

Au Cameroun, les travaux ethnographiques effectués par Ibrahim Mouiche (2011) sur le Mboscuda7 et par Patrice Bigombé Logo (1999) sur la Dynamique culturelle kirdi (DCK) soulignaient déjà la dimension ethnique des NMS. Dans leur sillon, les travaux effectués par le politologue Raymond Mbebi (2015) offrent des éléments pertinents pour comprendre et expliquer les expressions ethniques des NMS en contexte de démocratisation. Il s'agit du Ya-Kamata des Haoussa, l'Acgui des Guiziga à l'ExtrêmeNord, le Nguma-Mabi des Mabi au Sud, le Macuda des Mankon au Nord-Ouest et le Nkoul-Bebend des Maka-Bebend à l'Est. À ces exemples s'ajoutent d'autres NMS tels que le Ngondo des Sawa et le Moinam des Gbaya. Ces NMS ont des dimensions identitaires et des caractéristiques ethniques qu'ils mettent en avant pour produire une mobilisation capable de porter l'organisation. D'une part, ces NMS se forment sur la base d'une appartenance à une origine historique commune de ces membres. D'autre part, leurs membres sont liés par le partage de pratiques culturelles et politiques identiques.

Le Ya-Kamata est un NMS étudié pour la première fois par Mbebi (2011) et constitué des Haoussa, une ethnie appartenant à la civilisation soudanaise, laquelle s'étend des côtes guinéenne et sénégalaise au Nil, et du désert à la forêt. Les Haoussa se seraient déplacés de l'Afrique de l'Ouest vers le sud du continent jusqu'au Congo. Ils constituent par leur nombre, l'un des plus importants peuples d'Afrique (Nicolas, 1975: 400). Les travaux des auteurs comme Mizon (1895), Boutinot (1999) et Adama (2004) montrent que cette ethnie a migré et est entrée au Cameroun au début 19e siècle, par l'Adamaoua ;

6 Le projet de réforme du Code du travail porté par la ministre français du Travail, Myriam El Khomri en mars 2016.

7 Le MBOSCUDA est un NMS promu par la minorité ethnique Mbororo au Cameroun. 
puis au Sud du pays à la faveur du commerce des biens issus de l'élevage, de l'artisanat et de l'agriculture. Avec la libération politique qui intervient vers la fin des années 1980, accompagnée de l'ambition de créer un cadre d'expression culturelle et politique, la minorité ethnique haoussa va instituer le Ya-Kamata pour mieux se déployer dans l'espace public et acquérir du prestige. Le Ya-Kamata va ainsi œuvrer pour la promotion sociale, économique, culturelle des Haoussa au Cameroun. Le leader de ce NMS, El Hadj Oumarou, va faire de l'identité ethnique haoussa le socle de la construction d'une organisation capable d'accumuler des ressources politiques indispensables à son intégration politique.

L'Acgui (Association culturelle guiziga) est un NMS dont la formation s'est aussi opérée sur fond d'ethnicité. Ses membres sont tous des Guiziga, une ethnie présente au Nord du Cameroun, étudiée par Guy Pontié (1970) et tout récemment, par Mbebi (2015). Ses origines peinent à être retracées, mais se repèrent dans les mythes fondateurs des chefferies traditionnelles Lulu, Bi-Marva et Muturua (Fiéloux, 1975: 551-552). Pendant longtemps, les Guiziga ont été marginalisés, voire exclus des instances décisionnelles locales et nationales. La politique de l'équilibre régional doublée des microdosages intra-régionaux et locaux dans le Diamaré et le Mayo-Kani était à l'avantage des Peul, des Moundang et des Toupouri. À la faveur de la libéralisation politique et du multipartisme, les Guiziga mettront sur pied l'Acgui pour «briser leur marginalisation et accéder aux sphères de pouvoir » (Mbebi, 2015 : 100). Le fond ethnique de ce NMS se dégage nettement lorsque l'on observe ses principaux objectifs : la promotion de la culture guiziga, le développement du cadre socio-économique de la communauté guiziga, le renforcement des liens intra - et intercommunautaires.

Le Nguma-mabi des Mabi au Sud, le Macuda ${ }^{8}$ des Mankon au Nord-Ouest et le NkoulBebend des Maka-Bebend à l'Est sont d'autres NMS qui présentent également d'importantes assises ethniques et qui font de l'ethnicité une technologie de formation et d'action. La création du Nguma-mabi survient dans un contexte où les Mabi sont marginalisés, voire exclus des instances décisionnelles. Il s'agissait donc pour les élites Mabi d'engager à travers ce NMS l'émancipation et la représentation politiques des Mabi dans les appareils d'État (Mbebi, 2015 : 155). L'ethnie Mankon, quant à elle, avait réactivé une association déjà existante pour en faire un NMS dans le but d'avoir une représentation au gouvernement. Marginalisée, la communauté ethnique Mankon a profité de la libéralisation politique et du multipartisme pour transformer le Macuda en un NMS afin de se positionner stratégiquement sur l'échiquier politique national (Mbebi, 2015: 194). Enfin, les Maka-Bebend, à travers leur label ethnique, ont créé le Nkoul-Bebend pour s'investir dans les hautes sphères de l'État.

Ces illustrations issues des monographies anthropologiques montrent que le fait ethnique ne peut plus être considéré comme une donnée obsolète qu'il faut ignorer dans l'analyse de la formation des NMS. En effet, il est aujourd'hui évident que l'ethnicité est

8 MACUDA signifie Mankon Cultural And Development Association. 
une donnée sociale, culturelle et politique capable de produire des effets sur les mouvements sociaux. L'ethnicité apparaît aussi comme une ressource qui nourrit l'action des mouvements fondés sur l'ethnie. Parce qu'elle n'est pas toujours essentialiste c'està-dire immuable, elle est une donnée construite de l'organisation. C'est pourquoi nous récusons l'idée avancée par Guy Nicolas (1975) quand il affirme que le fait ethnique peut nourrir chez des individus la volonté de risquer leur vie, leur devenir ou de s'entretuer. En tout état de cause, l'existence d'une conscience ethnique dans la formation des NMS produit un discours sur l'ethnicité qui transcende celui sur la nation dans la mobilisation collective au Cameroun.

\section{Les discours mobilisateurs des NMS au Cameroun}

Tout discours nationaliste se particularise dans un contexte politique précis. En Afrique subsaharienne par exemple, durant l'accession à l'indépendance, le discours sur le nationalisme avait été très utile. En catalysant la mobilisation des indigènes, il avait alimenté la réponse sociopolitique à l'impunité de l'occupant raciste et à l'arrogance coloniale (McFadden, 2008 : 168). En faisant référence à l'histoire politique et constitutionnelle du Cameroun, on peut affirmer que les discours exaltant l'idée de la «République» sont louables en matière de démocratie. Les discours sur la nation constituent une rhétorique relativement idéaliste orientant les processus sociaux du système politique camerounais. Ainsi, lors de l'accession du Cameroun à l'indépendance, certains leaders politiques s'étaient montrés très attachés à la promotion de la nation comme unité symbolique de l'État en construction. Ces leaders politiques avaient alors diabolisé l'ethnicité en le pointant du doigt comme un obstacle au même titre que certains pouvoirs traditionnels (Nicolas, 1975 : 1018). La tyrannie de l'ère du temps durant cette période faisait du nationalisme une ressource capable de booster la mobilisation des Camerounais dans la poursuite du développement. La propagande nationaliste avait alors atteint son plus haut niveau dans la constitution du 2 juin 1972, puis dans la révision constitutionnelle du 18 janvier 1996. Il fallait à tout prix construire «la nation et l'identité nationale comme le veau d'or de toute société contemporaine» (Sindjoun, 1996:10).

Cependant, il faut souligner que la matrice agissante du discours nationaliste semble depuis peu sombrer dans une lente évanescence après avoir fait l'objet d'une histoire pleine d'agitations. Au regard des forces instituantes qui animent les mobilisations dans les NMS, on constate que ce discours recule. Il ne disparaît pas, mais perd en crédibilité comme nous le verrons plus bas. Sa force de mobilisation est aujourd'hui réduite et s'exprime de manière restreinte dans les incantations symboliques des institutions idéologiques de l'État. Concomitamment, de nombreux acteurs politiques camerounais, par un "usage politique du droit», ont procédé à une valorisation et à une «spectacularisation » de l'élément ethnique justifiant ainsi l'originalité de leur sys- 
tème politique (Njoya, 2002 : 241). Qui plus est, l'entame du processus de démocratisation depuis les années 1990 a changé la donne sur l'utilité primordiale du nationalisme. Dorénavant pour mobiliser, le pouvoir, par les discours saturés de non-dits, d'ellipses et de contradictions, exacerbe, au détriment du discours nationaliste, les particularismes sociaux en usant au besoin des appareils idéologiques de socialisation (Njoya, 2002 : 240). On assiste dès lors à l'éviction du discours national par le discours ethnique dans la réalisation des NMS.

\subsection{Crise du discours nationaliste dans la mobilisation des NMS}

Les limites du discours nationaliste au Cameroun ne s'objectivent que dans la conception subjective de la nation, celle du "vouloir vivre collectif» (Renan, 1882). Avec la démocratisation et la montée en puissance des NMS, ce discours n'est plus approprié pour former et mobiliser les groupes et les individus. En réalité, ce n'est pas tant le discours en lui-même qui pose problème, mais c'est son environnement d'énonciation qui ne lui est plus favorable. Plusieurs raisons expliquent cette réalité : dans cette société évoluant selon «la dialectique du local et du global» (Dimitrova, 2005: 6), il n'existe véritablement pas un sentiment d'appartenance des multiples ethnies à une même structure traditionnelle. L'ethnie béti, par exemple, ne se reconnaît pas dans les structures traditionnelles haoussa ou peuls. Ces derniers regardent, quant à eux, et avec une certaine étrangeté, les pratiques traditionnelles bamilékés (et réciproquement.). En plus du manque de continuité des traditions, le Cameroun est dépourvu d'institutions communes au sens culturel du terme (les institutions religieuses et linguistiques) capables de produire un esprit d'attachement pertinent ou une culture nationale relativement homogène.

Le discours nationaliste arrive difficilement à tirer profit de la laïcité du Cameroun pour amorcer une mobilisation sociale. Ici, la présence des religions traditionnelles reste aussi vivace que celle des religions révélées, c'est-à-dire la religion chrétienne avec ses divisions et l'islam (Momo, 1999: 824). Au Maroc par exemple, avant les années 2000 , le discours nationaliste ne mobilisait qu'une partie des mouvements intellectuels, alors que le sentiment religieux restait le moyen privilégié par ces mêmes intellectuels pour mobiliser les masses (Hajii, 1983; Rachik 2003 : 43-59). L’International Crisis Group, dans son rapport Afrique $n^{\circ} 229$, paru le 3 septembre 2015, pourtant consacré à la menace du radicalisme religieux affirme :

II existe au Cameroun une mosaïque d'un millier d'organisations religieuses dont 47 légalement enregistrées se répartissant entre chrétiens, musulmans et croyances traditionnelles 9 . Les chrétiens représentent $63 \%$ de la population, les musulmans $22 \%$, les religions traditionnelles ou animistes $14 \%$ et les courants gnostiques $1 \%$. Les musulmans sont majoritairement présents au Nord et dans

9 Communiqué radio presse $n^{\circ}$ 77/CRP/MINATD/DAP/SDLP, Ministère de l'administration territoriale et de la décentralisation (MINATD), 11 juillet 2008. 
la ville de Foumban à l'Ouest, mais on retrouve également d'importants foyers musulmans dans des villes au Sud telles que Douala et Yaoundé. Les chrétiens sont majoritaires au Sud. Les animistes se trouvent dans toutes les régions du Cameroun, mais ils sont plus présents à l'Ouest et dans la partie septentrionale. Cette répartition géographique des religions est le résultat de la loi du 19 décembre 1990 (International Crisis Group, 2015 : 1).

Ainsi, mobiliser par le discours nationaliste dans le contexte d'une multiplicité religieuse a toujours été très difficile. En outre, depuis les années 1960, le paysage religieux camerounais s'est complexifié. Cette configuration du champ religieux qui était ancien (avant et pendant la colonisation) et binaire (musulmans/chrétiens) a cédé la place à un paysage pluriel et diversifié. L'islam soufi est concurrencé par l'émergence du fondamentalisme wahhabite et la pénétration de courants radicaux (O’Brien, 1981). Pour le cardinal nigérian Francis Arinze (1991), au sein du christianisme, depuis 25 ans, de nouveaux mouvements religieux émergent, en particulier les églises néo-pentecôtistes dites «born again ». Ces nouveaux courants s'opposent à l'Église catholique, qui perd du terrain (Rosny, 2004 : 89). Le discours religieux devient alors une force complice de celui ethnique dans l'éviction du discours nationaliste. C'est pourquoi, à côté de la survie des religions " traditionnelles ", des progrès de l'islam, de la permanence du christianisme et des chrétientés d'origine missionnaire, le tableau religieux de l'Afrique noire aux XIX ${ }^{\mathrm{e}}$ et $\mathrm{XX}^{\mathrm{e}}$ siècles présente une prolifération remarquable de NMS (Moniot, 2005 : 382). Le fait religieux, tout en limitant le discours nationaliste, s'inscrit aux côtés du discours ethnique dans la structuration des NMS. Il n'est pas directement mis en avant dans le marché des mobilisations, mais plutôt utilisé de manière silencieuse dans les jeux symboliques par les activistes de mouvements.

Cette crise du discours nationaliste dans la mise en action des NMS est aussi travaillée par la pluralité linguistique du Cameroun. La langue est un moyen de communication structurée pour le service de la communauté ethnique qui l'utilise (Malherbe, 2004). Elle constitue parfois la principale identité du groupe (Bonte, Izard, 1991 : 410). Par conséquent, pour Patrice Gourdin (2015), la langue contribue à la nationalité ou à l'ethnicité et constitue un signe distinctif. Elle relie également des pans de l'histoire des populations, car le vainqueur, le conquérant, l'unificateur ou le colonisateur affirment leur pouvoir en diffusant leur langue, voire en l'imposant. Et sur ce point, la réalité camerounaise qui compte environ 268 langues semble se rapprocher du couple «langue/ethnie» au détriment du rapport «langue/nation». D’après Greenberg (1963), les langues camerounaises procédaient d'un enchevêtrement de langues des familles nigéro-congolaises, nilo-sahariennes, bantoues et chamito-sémitiques. Cependant, administrativement la population camerounaise est considérée comme bilingue : elle est majoritairement francophone (environ $80 \%$ de la population), mais compte une minorité (de quelque $20 \%$ ) d'anglophones. Le Cameroun applique dans ce contexte la formule de la division territoriale des langues coloniales. Les régions du nord-ouest et du sud-ouest constituent la portion anglophone autrefois sous administration britannique ; les huit autres régions forment la partie dite francophone (Noumssi, 2009). Cela 
dit, à part l'usage de l'anglais et du français, les langues traditionnelles parlées constituent donc un enjeu de politique interne essentiel dans la mobilisation des groupes et des individus au Cameroun. Et sur cet aspect, il est important de souligner que durant l'année 2016, l'on a vu émerger des mouvements sociaux dans cette partie dite anglophone du Cameroun et donc le fonds des revendications portait sur l'utilisation de l'anglais dans les institutions publiques.

En outre, le discours nationaliste porte en lui les erreurs du passé. Sur le plan historique au Cameroun, il a toujours été porteur de références aux multiples drames qui ponctuèrent le processus de la décolonisation et de constitution d'un centre politique à Yaoundé : brimades, emprisonnements, mutations des fonctionnaires soupçonnés de sympathies pour les idées de l'UPC ${ }^{10}$, licenciements, agressions contre des responsables ou cadres du parti «frappés, blessés et laissés pour morts dans la rue» (Um Nyobé, 1984). Le discours nationaliste historique garde les stigmates de la répression coloniale : dispersion des meetings par la police, arrestations, incendie de permanences de I'UPC, perquisitions dans les bureaux, exclusion des représentants du parti des commissions de révision des listes électorales, violation des correspondances (Mbembe, 1986:51). Ce dossier noir entache ce discours fondé sur la nation et l'empêche d'être capable de mobiliser aujourd'hui au même titre que le discours ethnique.

\subsection{La force du discours ethnique dans la dynamique des NMS}

Le discours ethnique, contrairement à son pendant nationaliste, est fondé sur les ethnies, qui sont de véritables unités anthropologiques en Afrique. L'ethnicité sur laquelle ce discours s'appuie est devenue une technologie d'élaboration des idéologies de mobilisation et d'affrontement (Courade, Sindjoun, 1996: 14). Si la production du discours est à la fois contrôlée, sélectionnée, organisée et redistribuée par un certain nombre de procédures qui ont pour rôle d'en conjurer les pouvoirs et les dangers (Foucault, 1970 : 3), au Cameroun, cette production dans les NMS a été à un moment donné une technologie incontrôlable. En effet, entre 1990 et 1992, elle avait donné lieu à des affrontements violents entre groupes ethniques Arabes Choa et Kotoko dans l'Extrême-Nord, entre Baya et Foulbé dans l'Adamaoua, pour modifier des rapports politiques inégaux précoloniaux (Courade, Sindjoun, 1996 : 7). De 1990 à 1995, dites « années de braises » au Cameroun en raison de la crise politique que connaît le pays suite aux revendications démocratiques, les grandes villes vont être le lieu de production d'énoncés à caractère discriminatoires vis-à-vis de ceux que l'on considère comme des allogènes. Dans un ouvrage ${ }^{11}$ publié en 1992 à Yaoundé, on retrouve des discours tels que :

10 L'Union des populations camerounaises est un parti politique historique qui a œuvré pour la décolonisation du Cameroun.

11 Voir Collectif «Changer le Cameroun» 1992, Le Cameroun éclaté ? Une anthologie commentée des revendications ethniques, Yaoundé, Editions C3. 
Go home [...] hors de nos terres. Bamenda-Bamiléké, [...] peuple beti, nous les fiers guerriers de la forêt équatoriale, ensemble, levons-nous pour anéantir l'avancée impérialiste des Bamiléké, car il y va de notre survie culturelle : chacun à sa place et nous sommes chez nous !

Par conséquent, l'élection d'élus locaux dans certaines métropoles du Cameroun avait entraîné des soulèvements des populations dites autochtones qui s'opposèrent à la gestion de leurs collectivités locales par des maires d'une origine ethnique autre que celle de leur territoire. C'est dans cette logique que les discours ethniques vont alimenter des NMS à caractère contestataire comme l'Essingang, le Laakam, l'organisation de libération du peuple sawa, le Front de libération du peuple beti et les groupes dits «anglophones ». On parle alors du retour de l'ethnicité dans le champ social et politique camerounais ou de l'ethnicisation des rapports sociaux (Courade, Sindjoun, 1996: 8).

Conscients de la force que peut avoir le discours ethnique, les acteurs sociaux et politiques vont s'atteler à la maîtrise de cette ressource pour atteindre leurs objectifs dans l'environnement libéral qu'offre le processus de démocratisation. L'ethnicité va désormais apparaître aux yeux des activistes de mouvements comme un produit de l'État et un mode de partage et d'appropriation de celui-ci, plutôt que sa négation ou sa subversion. Elle sera outre inséparable du processus de territorialisation humaine, politique et économique dont sa formation a été le vecteur depuis un siècle (Bayart, Geschiere, al., 2001: 180). Grâce au discours ethnique, les NMS traditionnellement apolitiques et non-lucratifs vont apparaître comme des structures utiles. D'une part, ils vont œuvrer pour la socialisation culturelle de leurs ressortissants se trouvant dans les villes métropolitaines ; d'autre part, ils seront le lieu où se négocient les demandes sociales concernant les membres de la communauté et l'endroit privilégié de la circulation de l'information. Les NMS, par le discours qui les anime, serviront de relais entre les membres de la communauté vivant en milieu urbain et ceux restés dans les zones rurales.

Cette analyse nous conduit au cœur de la dimension éminemment symbolique du discours ethnique. Un discours qui nourrit certes l'action, mais qui relève davantage du domaine du pouvoir symbolique que de l'organisation concrète. Il est emblème, étendard, slogan, pôle de référence, insigne, union, cri de ralliement, de révolte et appel aux armes à la fois. Il permet de donner un sens au champ de forces au sein duquel s'inscrit l'activiste du mouvement social. Ainsi, il apparaît toujours comme le cadre ultime le plus propice à une réaction à la contrainte politique. La référence à l'ethnie dans le discours mobilisateur confère aux tendances sociales, économiques et politiques une résonance et une puissance qu'elles ne détiennent pas par elles-mêmes (Nicolas, 1975: 1025-1026). Parallèlement, on observe le choix des symboles ethniques par les élites comme base de mobilisation pour appuyer leurs propres prétentions aux positions de prestige et aux avantages économiques dans une situation de compétition avec d'autres élites. Cela conduit souvent à une sorte de violence symbolique dans la rhétorique du discours mobilisateur. Quoi qu'il en soit, le discours ethnique des NMS consacre l'idée selon laquelle chaque groupe doit pouvoir défendre ses intérêts. Les candidats déclarés ou potentiels aux postes de commandement, les individus membres de la 
classe dirigeante viennent ici affirmer leur appartenance au groupe ethnique en même temps qu'ils demandent le soutien du mouvement social. Celui-ci devient ainsi un lieu de confrontation dont le vainqueur sort gratifié et rassuré par la reconnaissance dont il a bénéficié de la part des siens. Cet accord tacite entre le candidat et les représentants de la communauté peut être déterminant dans la compétition avec les autres prétendants au pouvoir puisque ces organisations peuvent jouer un rôle important dans la mobilisation des électeurs au profit d'un candidat (Balde, 2007:15).

Tout cela explique en partie le positionnement de certains leaders de mouvements à des postes importants au sein de l'appareil de l'État. C'est le cas par exemple de la nomination de Bakari Robert, l'un des leaders de l'Acgui, au prestigieux poste de délégué du gouvernement auprès de la communauté urbaine de Maroua, le 6 février 2009, d'Ayang Luc, président du Conseil économique et social, de Jean-Baptiste Baskouda, sénateur ${ }^{12}$, de Zacharie Pérevet, ministre, de Denis Oumarou, Secrétaire d'État, de Tikela Kemone, ancien conseiller spécial du chef de l'État et de François-Xavier Darwé, ancien directeur au ministère de l'élevage, tous membres très actifs de la Dynamique culturelle kirdi (Mbebi, 2015: 31-32). Leur discours s'est toujours inscrit dans une logique de mobilisation visant à décrier leur situation de marginalisés dans la gestion des affaires publiques, soit au niveau local, soit à l'échelle nationale. De par leurs discours, les acteurs des NMS reproduisent ainsi les clivages communautaires au Cameroun afin de créer des situations où émergent des allégeances, qui deviennent exploitables plus tard lors des compétitions politiques pour des fonctions de représentation. Des mots d'ordre sont généralement lancés pour orienter le choix politique des membres. C'est le cas très illustratif du président national du Ya-Kamata, El Hadj Oumarou qui occupe le poste de maire de la commune de Nkongsamba Ier depuis 2004. Ce «big man $»^{13}$ haoussa a investi ses ressources matérielles et son capital symbolique dans ce NMS afin de bénéficier de son appui lors des élections locales pour sa candidature dans la commune. Plus loin, le discours ethnique s'est aussi fait sentir dans le positionnement local de Mba Mba Grégoire au poste de maire grâce à la mobilisation du Nguma-Mabi. Aux élections municipales de 2002, il avait sollicité le soutien des chefs traditionnels mabi pour qu'ils le présentent comme le candidat du Nguma-Mabi. Ce qui se fit sans difficulté puisqu'il avait déjà l'estime des Mabi. Quelques mois avant la tenue de ces élections, Mba Mba Grégoire recevait alors solennellement l'onction communautaire des Mabi par les responsables du Nguma-Mabi à Elabé Marine (un hôtel à Kribi) (Mbebi, 2015 : 182-183).

Selon Mbebi (2015), les expressions du discours ethnique dans les NMS au Cameroun, viennent renforcer l'idée de Carolle Simard (2003) selon laquelle, il existe un lien entre

12 II faut souligner qu'au Cameroun, $70 \%$ des sénateurs sont élus par les citoyens et $30 \%$ sont nommés par le Président de la République. Voir, le décret № 2013/149 du 08 mai 2013 portant nomination de Sénateurs.

13 Le «big man » est une personne que les gens suivent dans la mesure où elle peut les procurer des bénéfices (Mouiche, 2005 : 122). 
les élus ethniques et les NMS. En effet, les élus ethniques s'attendent toujours à recevoir un appui politique d'une bonne partie de leur communauté d'origine. Cette attitude de proximité est particulièrement visible lors des campagnes électorales. Conscients des propriétés ethnoculturelles de leur circonscription électorale, les élus ethniques énoncent un discours approprié pour mobiliser les NMS dans la mesure où ces associations se composent d'électeurs de la même origine qu'eux, et ce notamment afin de contrer les effets négatifs du vote anti-ethnique d'une partie de l'électorat. Pour ce faire, ils travaillent en étroite collaboration avec les organismes communautaires. Le cas échéant, ils s'engagent à défendre et à promouvoir les intérêts des groupes ethniques présents dans leur circonscription électorale. L'attitude de proximité pousse la plupart des élus à se voir comme une courroie de transmission entre leur communauté d'origine, le mouvement social promu et la «machine municipale» (Simard, 2003 : 67).

\section{Conclusion}

Cette étude avait pour objectif principal de montrer que les NMS au Cameroun, et partant en Afrique subsaharienne, sont en grande partie des organisations qui ne peuvent être saisies sans la prise en compte de la variable ethnique. Sur le plan historique, nous avons souligné le fait que l'ethnicité était déjà mobilisée dans les mouvements indépendantistes que l'on croyait pourtant nationalistes à la fin de la période coloniale (1945-1960). Par la suite, l'ethnicité s'est retrouvée valorisée dans la formation des NMS durant la libéralisation politique dans les années 1990. Il s'agissait pour certaines minorités ethniques en position marginale de se constituer en mouvements capables de peser sur l'espace public afin d'intégrer le champ politique devenu réceptif aux revendications culturelles (Mouiche, 2012; Mbebi, 2015). Dès lors, face à cette prééminence de la variable ethnique dans les mobilisations, le discours nationaliste connaît une crise d'efficacité et d'action. Les tenants d'un discours fondé sur la nation arrivent difficilement à mobiliser les groupes et les individus à l'intérieur du pays. Certes, il est vrai que, au sein de la diaspora camerounaise, on peut déceler certaines formes de mobilisations fondées sur l'appartenance à la nation camerounaise en construction ${ }^{14}$. Mais ces mobilisations n'ont pas encore atteint un degré de sophistication semblable à celui des NMS en raison de leur faible structuration. Qui plus est, avec le pluralisme religieux et linguistique, le discours nationaliste sur les mobilisations des NMS semble s'étouffer dans une bulle. Il ne convainc presque plus personne et déçoit davantage les leaders de mouvements qui essayent de l'employer. De l'autre côté, le discours ethnique gagne en puissance et est davantage productif. En employant la catégorie d'ethnie dans ses énonciations, les activistes des NMS se garantissent une adhésion des membres de la communauté ethnique aux valeurs et idées qu'ils prônent. Dès lors, l'analyse des

14 C'est le cas des mobilisations panafricanistes, politiques, culturelles et artistiques observés dans certains pays d'Europe ou d'Amérique du Nord. 
NMS en Afrique noire sous le prisme de l'ethnicité ouvre de nombreuses perspectives de recherches pouvant être explorées à l'instar des modes d'appropriations des revendications des NMS par l'État, l'influence de la mondialisation sur les modes de formations et d'action des NMS, la portée de l'action sociale d'un NMS fondé sur l'ethnicité ou encore les dérives oligarchiques au sein de ces NMS.

\section{Bibliographie}

ADAMA H. (2004), L'islam au Cameroun : entre tradition et modernité, Paris, Harmattan.

AKINDES F. (2003), « Le lien social en question dans une Afrique en mutation », in J. BouLAD-Ayoub, L. BonNEville (dir.), Souverainetés en crise, Québec, Harmattan et Les Presses de l'Université Laval (Mercure du Nord), p. 379-403.

ARCAND S. (2003), Les minorités ethniques et l'État québécois: participation et représentations sociales des associations de groupes ethniques minoritaires lors de commissions parlementaires, 1974-2000, Thèse de doctorat en sociologie, Montréal, Université de Montréal.

ARINZE F. (1991), « Le défis des sectes et nouveaux mouvements religieux : une approche pastorale », L'observatoire Romano, 26 avril 1991.

BALDE A. (2007), « L'ethnie à l'épreuve des enjeux politiques: Réflexions sur le cas guinéen » Communication à la Conférence Internationale Statistiques sociales et diversité ethnique doit-on compter, comment et à quelles fins ?, Montréal, Organisée par le CIQSS et l'INED.

BAYART J. F., GesChIERE P., NYAMNJOH F. (2001), « Autochtonie, démocratie et citoyenneté en Afrique », Critique internationale, nº 10, p. 177-194.

BigombE Logo P. (1999), « Changement politique et dynamiques d'instrumentalisation de l'ethnicité Kirdi : l'ingénierie ethnopolitique », in L. Sindjoun (dir), La révolution passive au Cameroun : État, société et changement, Dakar, CODESRIA, p. 230-268.

BILOA E. (2003), L'influence du français sur l'anglais camerounais, consulté en ligne le 06 Décembre 2016. URL : http://www.sudlangues.sn/spip.php?article55

BITjAA Kody Z. D. (2007), «Enjeux politiques et territoriaux de l'usage du français au Cameroun », Hérodote, vol. 3, n 126, p. 57-68.

BONTE P., IZARD M. (1991), Dictionnaire de l'ethnologie et de l'anthropologie, Paris, P.U.F.

Boutinot L. (1999), Migration, religion et politique au nord-Cameroun, Paris, Harmattan.

CAHEN M. (2000), «États et frontières en Afrique subsaharienne », in C. DuboIs, M. MICHEL, P. SOUMILLE (dir), Frontières plurielles, Frontières conflictuelles en Afrique subsaharienne, Paris, Harmattan, p. 319-333. 
CopANS J., BuijtenHUijs B. (1982), « Le mouvement social en Afrique : un mouvement perpétuel ? », politique africaine, $\mathrm{n}^{\circ}$ 8, p. 3-7.

Coquery-Vidrovitch C., Moniot H. (2005), L’Afrique noire, de 1800 à nos jours, Paris, PUF, (Nouvelle Clio).

Courade G., Sindjoun L. (1996), «Le Cameroun dans l'entre-deux: introduction au thème. » Politique Africaine, $n^{\circ}$ 62, p. 3-14.

Courage G., Sindjoun L. (1996), « Le Cameroun dans l'entre-deux », Politique africaine, $\mathrm{n}^{\circ}$ 62, p.3-14.

Della Porta D., Diani M. (2006), Social Movements: An Introduction, Oxford, Blackwell, $2^{\mathrm{e}}$ edition.

Dimitrova A. (2005), « Le « jeu » entre le local et le global : dualité et dialectique de la globalisation », Socio-anthropologie. En ligne, consulté le 12 Septembre 2016. URL : https://socio-anthropologie.revues.org/440

Dubet F. (1994), «L'action étudiante », Universités et villes, Paris, Harmattan.

DufOUR P., TRAISNEL C. (2009), « Aux frontières mouvantes des mouvements sociaux, ou quand les partis politiques s'en mêlent. Le cas du souverainisme au Québec », Politique et Sociétés, vol. 28, $n^{\circ}$ 1, p. 37-62.

ERIKSEN T. H. (1991), « The cultural Context of Ethnic Differences», Man, vol. 26, n 1, p.127-144.

Eyinga A. (1985), Démocratie de Yaoundé - syndicalisme d’abord (1944-1946), t. 1, Paris, Harmattan.

FEENBERG A. (2005), "Le mouvement écologiste et la politique technologique», in M. Lowy (dir), Écologie et socialisme, Paris, Édition Syllepse, p. 45-80.

FENTON S. (2010), Ethnicity : Racism, Class and culture, Cambridge, Polity.

Fieloux M. (1975), « Les Guiziga du Cameroun septentrional. L'organisation traditionnelle et sa mise en contestation », Cahiers d'études africaines, vol. 15 n 59, p. 551-552.

Foucault M. (1970), L'ordre du discours, Paris, Collège de France.

Gourdin P. (2015), Manuel de géopolitique, éd. Diploweb.com

GreEnberg H. (1963), The languages of Africa, La Haye-Paris, Mouton.

HAJJI A. (1983), « Le maillon perdu de l'histoire du mouvement national », Revue Da'wat al-Haq, Rabat (Ministère des Habous et des affaires religieuses).

HAMEL F. (2008), « Mouvements infirmiers, représentation professionnelle et conflits sociaux », Recherche en soins infirmiers, vol 2, $n^{\circ} 93$, p. 44-48. 
JACQUEMART A. (2011), Les hommes dans les mouvements féministes français (1870-2010) : sociologie d'un engagement improbable. Thèse de doctorat en Sociologie, Paris, Ecole des Hautes Etudes en Sciences Sociales.

JACQUiOT P. (2007), « Comparaison des processus de formation et de diffusion du mouvement écologiste en RFA et en France », Cahiers internationaux de sociologie, vol 2, $\mathrm{n}^{\circ} 123$, p. 217-244.

JALLOH A. (1976), « Regional integration in Africa: lessons frons the past and prospects for the future », Africa development, vol. $1, \mathrm{n}^{\circ} \mathrm{2}, \mathrm{p} .48-53$.

LAPEYRONNIE D. (1998), « Mouvements sociaux et action politique : existe-t-il une théorie de la mobilisation des ressources ? », Revue française de sociologie, vol. 19, p. 593-619.

LONSDALE J. M. (1996), « Ethnicité morale et tribalisme politique », Politique africaine, $\mathrm{n}^{\circ}$ 61, p. 98-115.

MALHERBE M. (2004), Les langages de l'humanité. Une encyclopédie des 3000 langues parlées dans le monde, Paris, Laffont.

MARTINELlo M. (1995), L'ethnicité dans les sciences sociales contemporaines, Paris, PUF.

Mbebi R. (2015), Nouveaux Mouvements Sociaux et intégration sociopolitique des minorités ethniques au Cameroun, Thèse de doctorat en science politique, Yaoundé, Université de Yaoundé II.

Mbembe A. (1986), « Pouvoir des morts et langage des vivants : Les errances de la mémoire nationaliste au Cameroun », Politique africaine, $n^{\circ} 22$, p. 37-72.

McFADDEN P. (2008), «Les limites du nationalisme : citoyenneté et État. (Le nationalisme comme idéologie anticoloniale: une contextualisation)», Tumultes, vol. 2, $\mathrm{n}^{\circ} 31$, p. 167-183.

MCSweEn, N. (2010), "Repenser l'analyse des mouvements sociaux africains », Recherches, $\mathrm{n}^{\circ} 32$, p. 1-18.

MEDARD J.-F. (1977), « L’État sous développé au Cameroun », L’Année africaine, p. 33-84.

MENTHONG H. L. (1998), « Vote communautaire au Cameroun : un vote de cœur, de sang et de raison », Politique africaine, $n^{\circ} 69$, p. 40-52.

Meyer S. D., WhitTIER N. (2002), Social Movements. Identity, Culture and the State, Oxford, Oxford University Press.

Mizon L. (1895), «Les royaumes Foulbés du Soudan central », Annales de géographie, vol. $16, \mathrm{n}^{\circ} 4, \mathrm{p} .346-368$.

Mомо B. (1999), «La laïcité de l'État dans l'espace camerounais », Les Cahiers de droit, vol. $40, \mathrm{n}^{\circ} 4$, p.8 21-847. 
Mouiche I. (2000), « Ethnicité et multipartisme au Nord-Cameroun », African Association of Political Science, vol 5, $n^{\circ}$ 1, p. 46-91.

Mouiche I. (2005), Autorités traditionnelles et démocratisation au Cameroun : entre centralité de l'État et logiques de terroir, Munster, Lit Verlag.

Mouiche I. (2011), « Democratisation and political participation of Mbororo in Western Cameroun ", Africa Spectrum, vol 2, ${ }^{\circ}$ 46, p. 71-97.

NiCOLAS G. (1972), « Crise de l'État et affirmation ethnique en Afrique noire contemporaine », Revue française de science politique, vol. 22, n 5, p. 1017-1048.

Nicolas G. (1975), «Les catégories d'ethnie et de fraction ethnique au sein du système social hausa », Cahiers d'études africaines, vol. 15, n59, p. 399-441.

NJOYA J. (2001), « La constitutionnalisation des droits de minorités au Cameroun: Usages politiques du droit et phobie du séparatisme », Verfassung und Recht in Übersee, $\mathrm{n}^{\circ}$ 34. p. 24-47.

NJOYA J. (2002), « Démocratisation, divergences ethniques et politisation de la pluralité au Cameroun », Revue Canadienne des Études Africaines, vol. 36, n 2, p. 239-280.

Noumssi G. M. (2009), Dynamique du français au Cameroun : créativité, variations et problèmes sociolinguistiques, Yaoundé, Université de Yaoundé I.

O’BRIEN D. C. (1981), « La filière musulmane : confréries soufies et politique en Afrique noire », Politique africaine, $\mathrm{n}^{\circ}$ 4, p. 7-30.

OlIVIER DE SARDAN J.-P. (1973), Cours d'initiation au développement de l'Afrique en mutation, INADES formation, les éditions du Flamboyant.

ONANA J. (2004), Le sacre des indigènes évolués. Essai sur la professionnalisation politique, Chennevières-sur-Marne, Dianoïa.

OTAYEK R. (2001). «L’Afrique au prisme de l'ethnicité : perception française et actualité du débat », Revue internationale et stratégique, $\mathrm{n}^{\circ}$ 43, p. 129-142.

PonTIE G. (1970), Les Guiziga du Cameroun septentrional : L'organisation traditionnelle et les formes de la contestation, thèse de doctorat, Paris, EPHE.

RACHIK H. (2003), Symboliser la nation. Essai sur l'usage des identités collectives au Maroc, Casablanca, Éditions Le Fennec.

RENAN E. (1882), Qu'est-ce qu'une nation ? Paris, Édition Milles et une nuit.

ROSNY E. D. (2004), «Étude paradigmatique des nouveaux mouvements religieux et philosophiques à Douala », in S. GILLE (dir), L'effervescence religieuse en Afrique, Karthala, p. 89-62. 
SIMARD C. (2003), « Les élus issus des groupes ethniques minoritaires à Montréal : perceptions et représentations politiques, une étude exploratoire », Politique et Sociétés, vol. 22, $\mathrm{n}^{\circ} 1$, p. 53-78.

SindJoun L. (1996), « Identité nationale et révision constitutionnelle du 18 janvier 1996 : Comment constitutionnalise-t-on le "nous" au Cameroun dans l'État post-unitaire?», Revue camerounaise de science politique, $\mathrm{n}^{\circ} 1, \mathrm{p} .10-24$.

Tarrow S. (2005), The New Transnational Activism, Cambridge, Cambridge University Press.

TOURAINE A. (1993), La voix et le regard : sociologie des mouvements sociaux, Paris, Seuil.

Uм Nyobe R. (1984), Le problème national kamerounais, Paris, Harmattan.

Von Morgen C. (1982), À travers le Cameroun du sud au nord, Paris, Publications de la Sorbonne.

WEBER M. (1978), Economy and society, t. 1, California, University of California Press. 